\title{
Abstracts
}

\section{Hetmanka and Mother: Representing the Virgin Mary in Modern Poland}

Marian devotion has long been a central component of Catholic spirituality, in part because the image of the Virgin has been accommodated effectively within so many diverse cultural contexts. In modern Poland, Marianism gained much of its power from the way it linked seemingly contradictory models of femininity together within a national (or even nationalist) worldview. Mary, the Queen of Poland, has been offered to the faithful as a model for conceptualising the feminine within the nation, a model which is flexible enough to endure because it rests on a basic dichotomy: on the one hand, Mary is a powerful, sometimes militant, protector of Poland; on the other, she is an exemplar of feminine domesticity. She provides an image of authority and power which ultimately (perhaps paradoxically) poses little challenge to traditional norms of femininity - indeed, she is frequently called upon to fortify those norms. Marianism thus provides some of the glue that helps hold together two otherwise distinct strains of Polish national thought, one focused on maintaining conservative gender relations and the other on attaining victory in the international realm.

\section{A Spanish Genocide? Reflections on the Francoist Repression after the Spanish Civil War}

This article considers whether the Franco regime pursued a genocidal policy against Republicans after the formal ending of hostilities on I April 1939. In post-war Spain, the primary mechanism for punishing Republicans was military tribunals. Francoist military justice was based on the assumption that responsibility for the civil war lay with the Republic: defendants were tried for the crime of 'military rebellion'. This was, as Ramón Serrano Suñer admitted his memoirs, 'turning justice on its head'. But although it was extremely harsh, postwar military justice was never exterminatory. The article stresses that the institutionalisation of military justice from 1937, following the arbitrary murders of I936, contributed to a relative decline in executions. Although the regime's determination to punish Republicans for 'military rebellion' inevitably led to the initiation of tens of thousands of post-war military investigations, only a minority of cases ended in execution. This was especially the case from January I940, when the higher military authorities ended the autonomy of military tribunals over sentencing. This reassertion of central control in January I 940 was part of a wider policy to ease the self-inflicted problem of prison overcrowding; successive parole decrees led to a substantial and permanent decrease in the number of inmates by 1945. Allied victory in the Second World War did not mark the beginning but the end of the process of bringing to a close mass military justice. 


\section{Anti-communism at Home, Europeanism Abroad: Italian Cultural Policy at the Venice Biennale, I948-I958}

Using the fine arts exhibition of the Venice Biennale as a case study, this article considers the role of the Italian national government's cultural policy in pursuing its key domestic and foreign concerns between I 948 and I958. These were, respectively, suppressing communism at home, and promoting Western European unification and Italy's role within it. By scrutinising their involvement at the Biennale, it aims to show the importance placed not only by the Italian Christian Democrats but also by their European counterparts on constructing the idea of a culturally integrated Western Europe as a vital complement to analogous economic and political initiatives.

\section{'In Our District, the State Is Secure': The East German Secret Police Response to the Events of 1989 in Perleberg District}

This article details the year I989 in the East German District Perleberg up to the fall of the Wall as reflected in the documents of the Ministry for State Security the Stasi. It seeks to introduce empirical evidence on the course of the revolution in the towns of East Germany, an area which has received much less scholarly attention than larger centres. The article argues that in this particular outlying district, the generally accepted key factors behind the revolution (regime implosion, the changing international situation and popular pressure) are valid, but would best be weighted away from the changing international situation to the advantage of the other two. Furthermore, the evidence from District Perleberg suggests that pervasive state control, rather than accommodation and limited spheres for manoeuvre, was the dominant feature in 1989 in East Germany.

\section{Extraits}

\section{Mère et Hetmanka: les représentations de la Vierge Marie en Pologne}

La dévotion mariale est un des piliers de la spiritualité catholique, sans doute parce que l'image de la Vierge s'est prêtée à l'appropriation dans les contextes culturels les plus divers. Dans la Pologne contemporaine, la dévotion mariale tire sa force de son aptitude à tenir ensemble des modèles de féminité en apparence contradictoire. Marie, Reine de la Pologne, a représenté pour les croyants un modèle de féminité nationale, assez flexible pour perdurer parce qu'il joue d'apparents paradoxes. D'un côté, Marie est une protectrice puissante et active de la Pologne. De l'autre, elle est aussi un exemple de féminité domestique et familiale. Elle fournit ainsi une image d'autorité et de pouvoir qui ne menace pas les définitions traditionnelles de la féminité, bien au contraire. Le marialisme fournit ainsi le ciment qui contribue à rapprocher deux courants distincts de la pensée nationale polonaise, l'un qui se préoccupe de maintenir le status quo des relations de genre, l'autre qui poursuit des fins de gloire nationale sur l'échiquier du monde.

\section{Un génocide espagnol? Réflexions sur la répression franquiste}

Cet article s'interroge sur la dimension génocidaire de la répression franquiste contre les Républicains après le ler avril I939 et la fin des hostilités. Dès lors, les tribunaux militaires furent chargés de la répression. La justice militaire franquiste était basée sur le principe de la culpabilité de la République dans le déclenchement de la guerre civile, et les inculpés furent donc poursuivis pour 'rébellion armée'. Comme Ramón Serrano Suñer l'a admis, c'était 
mettre la justice cul par dessus tête. Si elle fut terrible, cette répression ne fut pas pour autant génocidaire. L'institutionnalisation de la justice militaire après I937, suite aux exécutions arbitraires de I936, a contribué à une diminution des exécutions. Parmi les dizaines de milliers de Républicains alors poursuivis, une minorité de personnes furent exécutées. Cela fut particulièrement vrai à partir de janvier I940, lorsque les hautes autorités militaires mirent fin à l'autonomie de jugement des tribunaux militaires. Cette décision faisait partie d'une politique destinée à diminuer l'encombrement des prisons espagnoles, et plusieurs mesures collectives de liberté surveillée firent décroître le nombre de détenus jusqu'en I945. La victoire alliée dans le conflit mondial survint ainsi à la fin du processus de liquidation de la justice militaire, bien plus qu'elle ne le provoqua.

\section{Anti-communiste à l'intérieur, pro-européenne à l'extérieur: le radis de la politique culturelle italienne vu à travers la Biennale de Venise, I948-I958}

A travers les expositions des Beaux-Arts de la Biennale de Venise, cet article s'intéresse à la place tenue par la politique culturelle dans la poursuite des objectifs domestiques et internationaux des gouvernements italiens entre I948 et I958. A l'intérieur, il s'agissait d'éradiquer le communisme en Italie. Vers l'extérieur, de travailler à l'unification de l'Europe Occidentale et à l'intégration de l'Italie dans ce nouvel ensemble. En étudiant la Biennale, on peut percevoir l'importance que les chrétiens démocrates italiens et leurs homologues européens attachaient à l'idée d'une Europe Occidentale culturellement homogène, complément indispensable aux initiatives politiques et économiques.

\section{'Dans notre district, l'Etat est en sécurité': la police secrète Est-allemande et les évènements de 1989 dans le district de Perleberg}

Cet article revient sur les évènements de I989 dans le district de Perleberg (Allemagne de l'Est), tells qu'ils furent racontés par les documents de la Stasi. Il présente des éléments sur le déroulement de la révolution dans une petite ville Est-allemande, alors que les travaux sur ces évènements ont jusque là privilégié les grands centres urbains. Il semble que dans ce district, les facteurs invoqués habituellement pour expliquer la révolution soient bien valides, mais que la désagrégation du régime et la pression populaire y jouent un rôle plus important que les modifications de la situation internationale. De surcroit, le cas de ce district suggère que le poids d'un l'Etat ubiquiste était bien le fait marquant de l'Allemagne de l'Est de I989, relativisant ainsi les considérations sur la dictature des limites ou les pratiques d'accommodation.

\section{Kurzfassungen}

\section{Hetmanka und Mutter: Die Repräsentation der Jungfrau Maria im modernen Polen}

Marienverehrung ist seit langem ein zentraler Bestandteil katholischer Spiritualität, und dies liegt zum Teil darin begründet, dass das Bild der Jungfrau sich so hervorragend in viele verschiedene kulturelle Kontexte einfügte. Im modernen Polen hat der Marianismus viel von seiner Überzeugungskraft durch die Art gewonnen, in der er scheinbar widersprüchliche Modelle von Weiblichkeit mit einem nationalen (oder sogar nationalistischen) Weltbild verband. Maria, die Königin Polens, wurde den Gläubigen als ein Modell zur Konzeptualisierung des Weiblichen innerhalb der Nation vorgestellt. Dieses Modell ist flexibel genug, um zu überdauern, da es auf einer grundlegenden Dichotomie beruht: einerseits ist Maria eine mächtige, manchmal militante Beschützerin Polens; andererseits ist sie ein Beispiel 
weiblicher Häuslichkeit. Sie bietet ein Abbild von Autorität und Macht welches letztlich (und vielleicht paradoxerweise) kaum die traditionellen Normen der Weiblichkeit herausfordert. Tatsächlich wird sie oft angerufen, um diese Normen zu festigen. Der Marianismus liefert somit ein bindendes Element, das zwei ansonsten verschiedene Merkmale des polnischen Nationalismus zusammenfügt: das Bemühen, sowohl konservative Geschlechterbeziehungen zu bewahren, als auch sich in der internationalen Arena zu behaupten.

\section{Ein spanischer Völkermord? Betrachtungen zur frankistischen Repression nach dem Ende des Spanischen Bürgerkriegs}

Dieser Artikel untersucht, ob das Franko Regime nach dem offiziellen Ende der Feindseligkeiten am I. April 1939 eine Politik des Genozids gegen die Republikaner verfolgte. Militärtribunale waren im Nachkriegsspanien der bevorzugte Mechanismus für die Bestrafung der Republikaner. Die frankistische Siegerjustiz basierte auf der Annahme, dass die Verantwortung für den Krieg bei der Republik lag: den Angeklagten wurde ein 'militärischer Aufstand' vorgeworfen. Dies bedeutete, wie Ramón Serrano Suñer in seinen Meoiren eingestand, die 'Gerechtigkeit auf den Kopf zu stellen'. Doch obwohl die militärische Nachkriegsjustiz extrem hart vorging, war sie doch nicht genozidär. Der Artikel unterstreicht, dass die Institutionalisierung der Militärjustiz seit I937zu einem relativen Rückgang der Exekutionen, nach den willkürlichen Morden im Jahr 1936, beitrug. Trotz der Entschlossenheit des Regimes, die Republikaner wegen 'militärischen Aufstands' zu bestrafen, was unvermeidlich $\mathrm{zu}$ Zehntausenden von militärgerichtlichen Untersuchungen führte, endete nur ein Bruchteil der Anklagen mit Hinrichtungen. Dies war insbesondere der Fall seit Januar I940, als die oberen militärischen Behörden die Autonomie der Militärtribunale im Hinblick auf die Verurteilungen beendete. Die Wiedergewinnung der zentralen Kontrolle im Januar I 940 war Bestandteil einer umfassenden Politik, die darauf abzielte, das selbstgeschaffene Problem der Überfüllung der Gefängnisse zu lindern. Nachfolgende Amnestien führten bis I945 zu einem umfangreichen und permanenten Rückgang der Zahl der Inhaftierten. Der alliierte Sieg im Zweiten Weltkrieg war nicht der Beginn, sondern das Ende eines Prozesses der Abschaffung massenhafter Militärjustiz.

\section{Anti-Kommunismus zu Hause, Europäismus im Ausland: Italienische Kulturpolitik auf der Biennale in Venedig, 1948-1958}

Dieser Beitrag diskutiert die Rolle der Kulturpolitik der italienischen Regierung in der Verfolgung ihrer wichtigsten innen- und aussenpolitischen Ziele zwischen I948 und 1958. Die Biennale-Kunstausstellung in Venedig dient hierbei als Fallstudie. Die Ziele der Regierung waren die Unterdrückung des Kommunismus im Inland und die Förderung der westeuropäischen Integration sowie der italienischen Rolle in diesem Prozess. Die Untersuchung der Beteiligung der italienischen Christdemokraten an der Biennale zielt darauf $\mathrm{ab}$, die Bedeutung aufzuzeigen, die nicht nur sie, sondern auch ihre Schwesterparteien in Europa der Idee eines kulturell integrierten Europas als unverzichtbare Ergänzung zu den gleichzeitigen wirtschaftlichen und politischen Initiativen beimassen.

\section{'In unserem Bezirk ist der Staat sicher': Die Reaktion der ostdeutschen Staatssicherheit auf die Ereignisse von 1989 im Bezirk Perleberg}

Auf der empirischen Grundlage der Papiere des Ministeriums für Staatssicherheit (Stasi) beschreibt dieser Artikel den Verlauf des Jahres I989 im Bezirk Perleberg bis zum Fall der Mauer. Dabei soll der Ablauf der Revolution im kleinstädtischen Ostdeutschland aufgezeigt werden, der bis jetzt weniger durch die Wissenschaft beachtet wurde als derjenige in den 
grösseren Zentren. Es wird argumentiert, dass in diesem abgelegenem Bezirk die allgemein bekannten Hauptursachen für den Aufstand (die Implosion des Regimes, der Wandel der internationalen Situation, sowie der Druck aus der Bevölkerung) alle zutreffen, dass aber die internationale Komponente eine geringere Rolle spielte als die anderen beiden Ursachen. Des weiteren deutet das Quellenmaterial aus dem Bezirk Perleberg darauf hin, dass die umfassende Kontrolle durch den Staat das dominante Merkmal des Jahres 1989 in Ostdeutschland war, und weniger Anpassung und begrenzte Freiräume. 